This item was submitted to Loughborough's Research Repository by the author.

Items in Figshare are protected by copyright, with all rights reserved, unless otherwise indicated.

\title{
Mixing rates and limit theorems for random intermittent maps
}

PLEASE CITE THE PUBLISHED VERSION

http://dx.doi.org/10.1088/0951-7715/29/4/1417

PUBLISHER

(c) IOP Publishing Ltd \& London Mathematical Society

VERSION

AM (Accepted Manuscript)

LICENCE

CC BY-NC-ND 4.0

REPOSITORY RECORD

Bahsoun, Wael, and Christopher Bose. 2019. "Mixing Rates and Limit Theorems for Random Intermittent Maps". figshare. https://hdl.handle.net/2134/21030. 


\title{
MIXING RATES AND LIMIT THEOREMS FOR RANDOM INTERMITTENT MAPS
}

\author{
WAEL BAHSOUN ${ }^{\dagger}$ AND CHRISTOPHER BOSE*
}

\begin{abstract}
We study random transformations built from intermittent maps on the unit interval that share a common neutral fixed point. We focus mainly on random selections of Pomeu-Manneville-type maps $T_{\alpha}$ using the full parameter range $0<\alpha<\infty$, in general. We derive a number of results around a common theme that illustrates in detail how the constituent map that is fastest mixing (i.e. smallest $\alpha$ ) combined with details of the randomizing process, determines the asymptotic properties of the random transformation. Our key result (Theorem 1.1) establishes sharp estimates on the position of return time intervals for the quenched dynamics. The main applications of this estimate are to limit laws (in particular, CLT and stable laws, depending on the parameters chosen in the range $0<\alpha<1$ ) for the associated skew product; these are detailed in Theorem 3.2. Since our estimates in Theorem 1.1 also hold for $1 \leq \alpha<\infty$ we study a second class of random transformations derived from piecewise affine Gaspard-Wang maps, prove existence of an infinite $(\sigma$-finite) invariant measure and study the corresponding correlation asymptotics. To the best of our knowledge, this latter kind of result is completely new in the setting of random transformations.
\end{abstract}

\section{INTRODUCTION}

In recent years, a lot of attention has been given to examples of nonuniformly expanding (or nonuniformly hyperbolic) maps with neutral fixed points. It is well known that such models can exhibit a range of nonstandard dynamical/probabilistic behavior; they may be mixing, but display subexponential decay of correlations for Hölder observables, for example. Limit theorems such as CLT and stable laws can be derived within various classes depending on the strength of the intermittency around the fixed point.

The purpose of this paper is to investigate similar questions for random transformations whose constituent maps are drawn from an appropriate nonuniformly expanding family. In particular, we aim to understand how behavior of the random transformation depends on properties of the maps and the randomizing process. A brief synopsis of our findings is as follows: At the level of existence (or non-existence) of a finite invariant measure and the rate of correlation decay for sufficiently regular observables, the random dynamics are completely determined by the map with fastest relaxation, independent of the randomization. The same also holds for the dynamical CLT when the correlation decay is strong enough to

Date: February 14, 2016.

1991 Mathematics Subject Classification. Primary 37A05, 37E05.

Key words and phrases. Interval maps with a neutral fixed point, intermittency, random dynamical systems, decay of correlations, Central Limit Theorem, stable laws.

The second author is supported by a research grant from the National Sciences and Engineering Research Council of Canada. 
be summable. However, we find the randomizing process begins to play an explicit role at the next finer level of analysis, for example, in sharp correlation asymptotics for regular observables supported away from the fixed points, and in limit theorems taking the form of stable laws for the associated skew product. Overall, this analysis gives a coherent picture that is consistent with our intuition about how randomness interacts with the intermittency.

We will work in the following concrete setting. Let $(I, \mathfrak{B}(I), m)$ denote the measure space consisting of the unit interval $I=[0,1]$ with Borel $\sigma$-algebra $\mathfrak{B}(I)$ and $m=$ Lebesgue measure on $\mathfrak{B}(I)$. The first part of this paper will concentrate on randomized one-dimensional maps of Pomeau-Manneville type [15]. A well-known, simplified version of the PM maps is the family of so-called Liverani-Saussol-Vaienti maps [12]. Such systems have attracted the attention of both mathematicians and physicists (see [11] for a recent work in this area).

To set our notation, given a parameter value $0<\alpha<\infty$, define

$$
T_{\alpha}(x)= \begin{cases}x\left(1+2^{\alpha} x^{\alpha}\right) & x \in\left[0, \frac{1}{2}\right] \\ 2 x-1 & x \in\left(\frac{1}{2}, 1\right] .\end{cases}
$$

When $\alpha=0, T_{\alpha}$ is the doubling map. For $\alpha>0, x=0$ is a neutral fixed point for the map $T_{\alpha}$ which is consequently a nonuniformly expanding, piecewise $C^{\infty}$, monotone map of the interval (on two pieces).

It is well-known that $T_{\alpha}$ admits a finite ACIM with density $h_{\alpha}=O\left(x^{-\frac{1}{\alpha}}\right)$ for $x$ near zero when $0<\alpha<1$ (see Liverani, Saussol, Vaienti [12], for example) and an infinite, $\sigma$-finite ACIM with similar asymptotic near zero when $1 \leq \alpha<\infty$ (see Pianigiani [14] for this range). In fact, the argument in [12] shows that for $0<\alpha<1$, the density $h_{\alpha}$ is locally Lipschitz on $(0,1]$ as well as being continuous and integrable.

Now fix two parameters $0<\alpha<\beta<\infty$ and consider the random LSV transformation defined as follows.

$$
T=\left\{T_{\alpha}(x), T_{\beta}(x) ; p_{1}, p_{2}\right\}, \text { where }
$$

$p_{1}, p_{2}>0$ and $p_{2}=1-p_{1}$. The random transformation $T$ maybe viewed as a Markov process with transition function

$$
\mathbb{P}(x, A)=p_{1} \mathbf{1}_{A}\left(T_{\alpha}(x)\right)+p_{2} \mathbf{1}_{A}\left(T_{\beta}(x)\right)
$$

of a point $x \in I$ into a set $A \in \mathfrak{B}(I)$. The transition function induces an operator, $E_{T}$, acting on measures; i.e., if $\mu$ is a measure on $(I, \mathfrak{B})$,

$$
\left(E_{T} \mu\right)(A)=p_{1} \mu\left(T_{\alpha}^{-1}(A)\right)+p_{2} \mu\left(T_{\beta}^{-1}(A)\right) .
$$

A measure $\mu$ is said to be $T$-invariant if

$$
\mu=E_{T} \mu,
$$

and $\mu$ is said to be an absolutely continuous invariant measure if $d \mu=f^{*} d m$, $f^{*} \geq 0$. To study absolutely continuous invariant measures, we introduce the transfer operator (Perron-Frobenius) of the random transformation $T$ :

$$
\left(P_{T} f\right)(x)=p_{1} P_{T_{\alpha}}(f)(x)+p_{2} P_{T_{\beta}}(f)(x),
$$

where $P_{T_{\alpha}}, P_{T_{\beta}}$ are the transfer operators associated with the $T_{\alpha}, T_{\beta}$ respectively. Then it is a straight-forward computation to show that a measure $\mu=f^{*} \cdot m$ is an 
absolutely continuous $T$-invariant measure if

$$
P_{T} f^{*}=f^{*} \text {. }
$$

1.1. A skew product representation. Define the skew product transformation $S(x, \omega): I \times I \rightarrow I \times I$ by

$$
S(x, \omega)=\left(T_{\alpha(\omega)}, \varphi(\omega)\right)
$$

where

$$
\alpha(\omega)=\left\{\begin{array}{ll}
\alpha & , \omega \in\left[0, p_{1}\right) \\
\beta & , \omega \in\left[p_{1}, 1\right]
\end{array} \quad ; \quad \varphi(\omega)=\left\{\begin{array}{ll}
\frac{\omega}{p_{1}} & , \omega \in\left[0, p_{1}\right) \\
\frac{\omega-p_{1}}{p_{2}} & , \omega \in\left[p_{1}, 1\right]
\end{array} .\right.\right.
$$

The skew product representation in (1.1) is a version ${ }^{1}$ of the skew product representation which was studied in Bahsoun, Bose and Quas [5]. We denote the transfer operator associated with $S$ by $\mathcal{L}_{S}$ : for $g \in L^{1}(I \times I)$ and measurable $A \subseteq I \times I$,

$$
\int_{S^{-1} A} g d(m \times m)(x, \omega)=\int_{A} \mathcal{L}_{S} g d(m \times m)(x, \omega) .
$$

Then a measure $\nu$, such that $d \nu=g^{*} d(m \times m)$ and $\int_{I \times I} g^{*} d(m \times m)=1$, is an absolutely continuous $S$-invariant probability measure if

$$
\mathcal{L}_{S} g^{*}=g^{*} \text {. }
$$

In [5], Theorem 5.2 it is shown that if $g \in L^{1}(I \times I)$ and $\mathcal{L}_{S} g=\lambda g$ with $|\lambda|=1$, then

$$
g(x, \omega)=f(x) \cdot \mathbf{1}(\omega)
$$

and $P_{T} f=\lambda f$, that is, $g$ depends only on the spatial coordinate $x$ and as a function of $x$ only, is also an eigenfunction for $P_{T}$. Setting $\lambda=1$ we obtain $\mathcal{L}_{S} g^{*}=g^{*}$ if and only if $g^{*}(x, \omega)=f^{*}(x)$ with $P_{T} f^{*}=f^{*}$. Consequently there is a one to one correspondence between invariant densities for $S$ and invariant densities for $T$. Moreover, dynamical properties such as ergodicity, number of ergodic components or weak-mixing, properties that are determined by peripheral eigenfunctions, can be determined via either system.

Our skew product construction is similar to a model constructed by Gouëzel [8], however in that paper, the skew product samples continuously from the space of LSV maps, whereas we sample discretely. This allows us to simplify the analysis and extend the range of parameters in which we can complete the analysis, compared to [8]. A more detailed discusion and comparison between the two models can be found in Bahsoun, Bose and Duan [4].

1.2. Inducing for the skew representation $S$. The method of inducing (equivalently, Markov extensions or Young towers) gives a systematic way to study maps like $T_{\alpha}$ having localized singularities, for example, as detailed in Young [17]. We will begin by doing essentially the same thing with our skew product $S$, inducing on the right half of the square $\Delta_{0}:=(1 / 2,1] \times[0,1]$.

Set

$$
T_{\omega}^{n}(x):=T_{\alpha\left(\varphi^{n-1} \omega\right)} \circ \ldots \circ T_{\alpha(\varphi \omega)} \circ T_{\alpha(\omega)}(x) .
$$

\footnotetext{
${ }^{1}$ The results obtained in Bahsoun, Bose and Quas [5] are valid for any class of measurable non-singular maps on $\mathbb{R}^{q}$, without any regularity assumptions. Moreover in [5], the probability distribution on the noise space is allowed to be place-dependent.
} 
Then

$$
S^{n}(x, \omega)=\left(T_{\omega}^{n}(x), \varphi^{n}(\omega)\right)
$$

Also, set

$$
P_{\omega}^{n}:=p_{\alpha\left(\varphi^{n-1} \omega\right)} \times \ldots \times p_{\alpha(\varphi \omega)} \times p_{\alpha(\omega)},
$$

where $p_{\alpha(\omega)}=p_{1}$, for $\alpha(\omega)=\alpha$ and $p_{\alpha(\omega)}=p_{2}$, for $\alpha(\omega)=\beta$. We define two sequences of random points $\left\{x_{n}(\omega)\right\}$ and $\left\{x_{n}^{\prime}(\omega)\right\}$ in $[0,1]$ which will be used to construct the first return map of $S$ to $\Delta_{0}$. The points $x_{n}(\omega)$ lie in $(0,1 / 2]$. Set

$$
x_{1}(\omega) \equiv \frac{1}{2} \text { and } x_{n}(\omega)=\left.T_{\alpha(\omega)}^{-1}\right|_{\left[0, \frac{1}{2}\right]}\left[x_{n-1}(\varphi \omega)\right], n \geq 2 .
$$

Observe that with this notation,

$$
S\left(x_{n}(\omega), \omega\right)=\left(T_{\alpha(\omega)}\left(x_{n}(\omega)\right), \varphi \omega\right)=\left(x_{n-1}(\varphi \omega), \varphi \omega\right) .
$$

The points $x_{n}^{\prime}(\omega)$ lie in $\left(\frac{1}{2}, 1\right]$, defined by

$$
x_{0}^{\prime}(\omega) \equiv 1, x_{1}^{\prime}(\omega) \equiv \frac{3}{4} \text { and } x_{n}^{\prime}(\omega)=\frac{x_{n}(\varphi \omega)+1}{2}, n \geq 2,
$$

that is, the $x_{n}^{\prime}(\omega)$ are preimages of the $x_{n}(\varphi \omega)$ in $\left(\frac{1}{2}, 1\right]$ under the right branch $2 x-1$.

1.3. First return map of $S$ to $\Delta_{0}$. Let $R: \Delta_{0} \rightarrow \mathbb{Z}^{+}$be the first return time function and $S^{R}: \Delta_{0} \rightarrow \Delta_{0}$ be the return map. For $n \geq 1$, set $I_{n}(\omega):=$ $\left(x_{n+1}(\omega), x_{n}(\omega)\right]$ and $J_{n}(\omega):=\left(x_{n}^{\prime}(\omega), x_{n-1}^{\prime}(\omega)\right]$. Observe that every point in $J_{n}(\omega)$ will return to $\left(\frac{1}{2}, 1\right]$ in $n$ steps under the random iteration $T_{\omega}^{n}$ as follows:

$$
J_{n}(\omega) \rightarrow I_{n-1}(\varphi \omega) \rightarrow I_{n-2}\left(\varphi^{2} \omega\right) \rightarrow \ldots \rightarrow I_{1}\left(\varphi^{n-1} \omega\right) \rightarrow\left(\frac{1}{2}, 1\right] .
$$

Next, we partition $\Delta_{0}$ into subsets $\Delta_{0, i}, i=1,2, \ldots$ where

$$
\Delta_{0, i}:=\left\{(x, \omega) \mid x \in J_{i}(\omega)\right\}
$$

and then further partition each $\Delta_{0, i}$ into subsets $\Delta_{0, i}^{j}, j=1,2, \ldots 2^{i}$ according to the $2^{i}$ possible values of the string $\alpha(\omega), \alpha(\varphi \omega), \ldots \alpha\left(\varphi^{i-1} \omega\right)$. Defined this way, $S^{i}$ maps each subset $\Delta_{0, i}^{j}$ bijectively to $\Delta_{0}$.

For example, in the case $i=2$, there are four sets $\Delta_{0,2}^{j}$ on which $R=2$ and such that $S^{R}$ maps each set bijectively to $\Delta_{0}$ :

$$
\Delta_{0,2}^{j}= \begin{cases}J_{2}(\omega) \times\left[0, p_{1}^{2}\right), & \text { if } j=1, \\ J_{2}(\omega) \times\left[p_{1}^{2}, p_{1}\right), & \text { if } j=2, \\ J_{2}(\omega) \times\left[p_{1}, p_{1}+p_{1} \cdot p_{2}\right), & \text { if } j=3, \\ J_{2}(\omega) \times\left[p_{1}+p_{1} \cdot p_{2}, 1\right), & \text { if } j=4\end{cases}
$$

To summarize,

$$
\Delta_{0, i}=\bigcup_{j=1}^{2^{i}} \Delta_{0, i}^{j}
$$

and

$$
\Delta_{0}=\bigcup_{i=1}^{\infty} \bigcup_{j=1}^{2^{i}} \Delta_{0, i}^{j}
$$


where, for every $i$ and $j=1,2, \ldots, 2^{i}$,

$$
\left.R\right|_{\Delta_{0, i}^{j}}=i .
$$

For each $n$, the interval $J_{n}(\omega)$ depends on only the first $n$ coordinates in $\omega$ and moreover

$$
m \times m\{R=n\}=\sum_{j=1}^{2^{n}} P_{\omega_{j}}^{n} m\left(J_{n}\left(\omega_{j}\right)\right)=E_{\omega}\left(m\left(J_{n}(\omega)\right)\right),
$$

where $\omega_{j}$ ranges across the $2^{n}$ possible configurations $\omega$ with distinct values for the string $\alpha(\omega), \alpha(\varphi \omega), \ldots \alpha\left(\varphi^{n-1} \omega\right)$ and $E_{\omega}(\cdot)$ denotes expectation with respect to $\omega$. Since $m\left(J_{n}(\omega)\right)=\frac{1}{2} m\left(I_{n-1}(\omega)\right)$ we also obtain

$$
m \times m\{R>n\}=E_{\omega}\left(x_{n}^{\prime}(\omega)-1 / 2\right)=\frac{1}{2} E_{\omega}\left(x_{n}(\omega)\right) .
$$

Finally, we adopt the following (standard) notation throughout this paper. Given sequences $a_{n}$, (respectively $b_{n}$ ) of nonnegative (respectively positive) real numbers, we write $a_{n} \asymp b_{n}$ if there is a constant $C \geq 1$ such that $C^{-1} b_{n} \leq a_{n} \leq C b_{n}$, and $a_{n} \sim b_{n}$ if $\lim \frac{a_{n}}{b_{n}}=1$.

1.4. Statement of the main result in this paper. There is now a range of studies (including Young [17], Zweimüller [18, 19], Sarig [16], Gouëzel [7] and MelbourneTerhesiu [13], for example) that show how careful analysis of the asymptotics of $m\{R>n\}$ can reveal deep statistical properties of the underlaying map. In our case, we are interested in the skew $S$ acting on the square. The strength of our results, therefore, are likely to depend in a critical way, on the sharpness of estimates obtained on the measures of sets like $J_{n}(\omega)$ and $I_{n}(\omega)$.

For example, in [17] a key estimate for a single LSV-map $T_{\alpha}$ reads as follows: if $x_{n}$ is the sequence of points generated under the inverse of the leftmost branch of $T_{\alpha}$, such that $T_{\alpha} x_{n+1}=x_{n}$ and $x_{1}=1 / 2$, then there exists $c>0$ such that $c^{-1} n^{-\frac{1}{\alpha}} \leq x_{n} \leq c n^{-\frac{1}{\alpha}}$. If we introduce the notation $x_{n}(\alpha):=x_{n}$ for this sequence of deterministic points, we can record this observation as

$$
x_{n}(\alpha) \asymp n^{-\frac{1}{\alpha}}
$$

which upper bounds the size of return-time sets and is sufficient for establishing existence of the invariant density $h_{\alpha}$ and bounds on rate of correlation decay when $0<\alpha<1$ as detailed in [17]. In fact, the analysis in [17] actually proves more: define $c_{n}(\alpha):=n^{\frac{1}{\alpha}} x_{n}(\alpha)$. Then $x_{n}(\alpha)=c_{n}(\alpha) n^{-\frac{1}{\alpha}}$ with $\lim _{n} c_{n}(\alpha)=\frac{1}{2} \alpha^{-\frac{1}{\alpha}}:=$ $c(\alpha)$. That is, in our notation

$$
x_{n}(\alpha) \sim \frac{1}{2 \alpha^{\frac{1}{\alpha}}} n^{-\frac{1}{\alpha}}=c(\alpha) n^{-\frac{1}{\alpha}}
$$

This sharper estimate is key for analysis of limit theorems for maps like $T_{\alpha}$. See, for example, Melbourne and Terhesiu [13] and Gouëzel [9].

Moving to similar estimates on our skew product $S$, the following rough estimate is obtained as Lemma 4.4 in Bahsoun, Bose and Duan [4] as a first step in their analysis: For all $\omega \in[0,1]$ and $n \geq 1$

$$
x_{n}(\alpha) \leq x_{n}(\omega) \leq x_{n}(\beta)
$$


where $x_{n}(\beta)$ denotes the sequence of deterministic points for $T_{\beta}$. The main result in this paper is a much sharpened estimate on the location of $x_{n}(\omega)$ compared to Equation (1.8).

Keeping the bounds (1.8) in mind, and following the setup for equation (1.7), for each $n, \omega$ define $c_{n}(\omega):=n^{\frac{1}{\alpha}} x_{n}(\omega)$ (so that $x_{n}(\omega)=c_{n}(\omega) n^{-\frac{1}{\alpha}}$ ). We now state the main result in our paper:

Theorem 1.1. Let $0<\alpha<\beta<\infty$. For almost every $\omega$ in $[0,1]$ we have $\lim _{n} c_{n}(\omega)=\frac{1}{2}\left(\alpha p_{1}\right)^{-\frac{1}{\alpha}}=c(\alpha) p_{1}^{-\frac{1}{\alpha}}$. That is

$$
x_{n}(\omega) \sim c(\alpha) p_{1}^{-\frac{1}{\alpha}} n^{-\frac{1}{\alpha}} .
$$

Moreover, $E_{\omega}\left(\left|c_{n}(\omega)-c(\alpha) p_{1}^{-\frac{1}{\alpha}}\right|\right) \rightarrow 0$, in other words, convergence of $n^{\frac{1}{\alpha}} x_{n}$ to $c(\alpha) p_{1}^{-\frac{1}{\alpha}}$ also holds in the $L^{1}-$ norm.

In the terminology of random dynamical systems, this is a quenched limit theorem (ie: almost everywhere) as opposed to annealed (averaged over $\omega$ ). In general, quenched results are harder to obtain than annealed ones. Examples of other quenched limit theorems can be found in Ayyer, Liverani and Stenlund [3].

The significance of Theorem 1.1 is what it implies for asymptotics of the random system. The main applications of this theorem will appear in Sections 3 and 4 where we derive limit theorems for the skew product $S$ and study asymptotics for infinite measure preserving systems, respectively. However, to illustrate the flavour of our results in a simple context, we close this section by revisiting (and sharpening ${ }^{2}$ ) the main conclusion from [4] that shows one way in which the fast system $\left(T_{\alpha}\right)$ dominates the asymptotic behavior of the skew:

Theorem 1.2. Let $0<\alpha<\beta<1$ and $S$ be as defined in (1.1). Then $m \times m\{R>$ $n\} \sim \frac{1}{2} c(\alpha) p_{1}^{-\frac{1}{\alpha}} n^{-\frac{1}{\alpha}}=\frac{1}{4}\left(\alpha p_{1}\right)^{-\frac{1}{\alpha}} n^{-\frac{1}{\alpha}}$. Moreover,

(1) $S$ admits a unique absolutely continuous invariant probability measure $\nu$ with density $d \nu=h d(m \times m)$ where $h$ is Lipschitz on compact subsets of $(0,1] \times[0,1]$

(2) $(S, \nu)$ is mixing;

(3) for $\phi \in L^{\infty}(I \times I, m \times m)$ and $\psi$ a Hölder continuous function on $I \times I$

$$
|\operatorname{Cor}(\phi, \psi)|=O\left(n^{1-\frac{1}{\alpha}}\right),
$$

where

$$
\operatorname{Cor}(\phi, \psi)=\int \phi \circ S^{n} \cdot \psi d \nu-\int \phi d \nu \int \psi d \nu
$$

With more assumptions on the observables $\phi$ and $\psi$ we obtain the following stronger estimate:

(4) If $\phi \in L^{\infty}(I \times I, m \times m)$ and $\psi$ Lipschitz on $I \times I, \int \phi d \nu \neq 0$ and $\int \psi d \nu \neq 0$ with both $\phi$ and $\psi$ identically 0 in an open strip containing the line $x=0$, then

$$
\operatorname{Cor}(\phi, \psi) \sim \frac{1}{4} E_{\omega}\left(h\left(\frac{1}{2}, \omega\right)\right)\left(\alpha p_{1}\right)^{-\frac{1}{\alpha}}\left(\frac{1}{\alpha}-1\right)^{-1} n^{1-\frac{1}{\alpha}} \int \phi d \nu \int \psi d \nu .
$$

\footnotetext{
${ }^{2}$ Statement (3) in the current Theorem 1.2 is essentially proved in [4]. The exact asymptotics of $m \times m\{R>n\}$ however, are new, and the precise decay of correlations in (4) for functions supported away from the line $x=0$ are also new.
} 
Proof. The enumerated statements $(1-3)$ follow by identical arguments to those in [4] once the claimed asymptotics on $m \times m\{R>n\}$ are derived. The latter are easily established since by Equation (1.5) we know $m \times m\{R>n\}=\frac{1}{2} E_{\omega}\left(x_{n}(\omega)\right)=$ $\frac{1}{2} n^{-\frac{1}{\alpha}} E_{\omega}\left(c_{n}(\omega)\right)$, while Theorem 1.1 implies $E_{\omega}\left(c_{n}(\omega)\right) \rightarrow c(\alpha) p_{1}^{-\frac{1}{\alpha}} \in(0, \infty)$. It follows that $m \times m\{R>n\} \sim \frac{1}{2} c(\alpha) p_{1}^{-\frac{1}{\alpha}} n^{-\frac{1}{\alpha}}$. The fact that the density $h$ is Lipschitz on compact subsets of $(0,1] \times I$ is proved in Lemma 3.1 in Section 3 of this paper.

To establish (4) we first assume that $\phi, \psi$ are supported on $\Delta_{0}$ with the stated regularity. By Theorem 6.3 in Gouëzel [7] we have

$$
\operatorname{Cor}(\phi, \psi) \sim \sum_{k>n} \nu\{R>k\} \int \phi d \nu \int \psi d \nu
$$

The invariant measure $d \nu=h d m \times d m$, with $h$ Lipschitz on $\Delta_{0}$. This leads to the following estimate (see Lemma 3.3 in Section 3 ) on the measure of return time sets:

$$
\nu\{R>k\} \sim \frac{1}{4} E_{\omega}\left(h\left(\frac{1}{2}, \omega\right)\right)\left(\alpha p_{1}\right)^{-\frac{1}{\alpha}} k^{-\frac{1}{\alpha}} .
$$

Summing over $k>n$ gives the result.

Now using the argument from Gouëzel [7] Section 7 in our setting we can extend the support of $\phi, \psi$ to $\Delta_{N}:=\left\{(x, \omega): x_{N}(\omega) \leq x \leq 1\right\}$, with the same asymptotic return times. For sufficiently large $N$ this picks up the support of $\phi$ and $\psi$.

The rest of this paper is organized as follows. In Section 2 we prove Theorem 1.1. The computation depends on a classical result of Hoeffding [10] that gives exponental decay of large deviations for sums of bounded, independent random variables.

In Section 3 we apply our estimates to derive central limit theorems and stable laws for the Birkhoff sums

$$
S_{n} f(x, \omega):=\sum_{k=0}^{n-1} f\left(S^{k}(x, \omega)\right),
$$

where $S$ is the skew product and parameters $0<\alpha<\beta<1$ pass through various ranges leading to quantitatively different asymptotics. Precise results are itemized in Theorem 3.2. Finally, in Section 4 we discuss a related skew product system (of Gaspard-Wang-type maps, see [6], indexed by $0<\alpha<\infty)$. When $1 \leq \alpha<\beta$ a natural analogue of Theorem 1.1 applies, and the invariant measure is bound to be infinite $(\sigma$-finite). We investigate correlation asymptotics for this case. To the best of our knowledge this is the first detailed analyis of asymptotics for a random system with an infinite invariant measure.

\section{Proof of Theorem 1.1}

We begin with a basic calculus estimate.

Lemma 2.1. Let $0<\alpha<\infty$ and $0 \leq x \leq 1$. Then

$$
1-\alpha x \leq[1+x]^{-\alpha} \leq 1-\alpha x+\frac{\alpha(1+\alpha)}{2} x^{2}
$$

Proof. Elementary. 
Recall our notation $x_{n}(\alpha)$ (resp. $x_{n}(\beta)$ ) for the sequence of points generated by first branch inverse of the deterministic map $T_{\alpha}$ (resp. $T_{\beta}$ ). Recall also the basic estimates in Equations (1.6), (1.7) and (1.8). Our goal is to obtain sharp decay estimates on $n\left[x_{n}(\omega)\right]^{\alpha}$ and for that we will need the following classical large deviations estimate.

Proposition 2.2. (Hoeffding [10], Theorem 1) Suppose that $X_{k}=X_{k}(\omega), k=$ $1,2, \ldots n$ are independent random variables, uniformly bounded such that $0 \leq X_{k} \leq$ 1. Let $\bar{X}_{n}=n^{-1} \sum_{k=1}^{n} X_{k}$ and $E\left(\bar{X}_{n}\right)=n^{-1} \sum_{k=1}^{n} E\left(X_{k}\right)$. Then for every $t>0$ we have

$$
\mathbb{P}\left\{\left|\bar{X}_{n}-E\left(\bar{X}_{n}\right)\right| \geq t\right\} \leq \exp \left(-2 n t^{2}\right)
$$

We proceed by a sequence of lemmas.

Lemma 2.3. There is a set $G_{1} \subseteq[0,1]$ of full measure such that for every $\omega \in G_{1}$ we have

$$
\limsup n^{\frac{1}{\alpha}} x_{n}(\omega) \leq\left[\alpha 2^{\alpha} p_{1}\right]^{-\frac{1}{\alpha}}=c(\alpha) p_{1}^{-\frac{1}{\alpha}} .
$$

Proof. We begin with the standard expression derived directly from the definition of $T_{\alpha(\omega)}$ :

$$
\frac{1}{\left[x_{n-1}(\varphi \omega)\right]^{\alpha}}=\frac{1}{\left[x_{n}(\omega)\right]^{\alpha}}\left[1+\left[2 x_{n}(\omega)\right]^{\alpha(\omega)}\right]^{-\alpha} .
$$

Using the upper bound contained in the right hand side of Lemma 2.1, with $x=$ $\left[2 x_{n}(\omega)\right]^{\alpha(\omega)}$ and reordering terms we obtain

$$
\frac{1}{\left[x_{n}(\omega)\right]^{\alpha}}-\frac{1}{\left[x_{n-1}(\varphi \omega)\right]^{\alpha}} \geq \alpha 2^{\alpha}\left[2 x_{n}(\omega)\right]^{\alpha(\omega)-\alpha}-\frac{\alpha(1+\alpha)}{2} 2^{\alpha}\left[2 x_{n}(\omega)\right]^{2 \alpha(\omega)-\alpha} \text {. }
$$

Applying this inequality along the sequence $x_{k}\left(\varphi^{n-k} \omega\right)$ for $k=2$ through $n$, and keeping in mind that $x_{1}(\omega)=\frac{1}{2}$ for every $\omega$ gives the basic inequality

$$
\begin{aligned}
\frac{1}{\left[x_{n}(\omega)\right]^{\alpha} \geq 2^{\alpha}}+\alpha 2^{\alpha}\left\{\sum_{k=2}^{n}\left[2 x_{k}\left(\varphi^{n-k} \omega\right)\right]^{\alpha\left(\varphi^{n-k} \omega\right)-\alpha}\right. \\
\left.-\frac{1+\alpha}{2} \sum_{k=2}^{n}\left[2 x_{k}\left(\varphi^{n-k} \omega\right)\right]^{2 \alpha\left(\varphi^{n-k} \omega\right)-\alpha}\right\} .
\end{aligned}
$$

Next, we use the estimate contained in Equation (1.8), the notation from Equation (1.7) and division by $n$ to obtain

$$
\begin{aligned}
\frac{1}{n\left[x_{n}(\omega)\right]^{\alpha} \geq \frac{2^{\alpha}}{n}} & +\frac{n-1}{n} \alpha 2^{\alpha}\left\{\frac{1}{n-1} \sum_{k=2}^{n}\left[\frac{2 c_{k}(\alpha)}{k^{\frac{1}{\alpha}}}\right]^{\alpha\left(\varphi^{n-k} \omega\right)-\alpha}\right. \\
& \left.-\frac{1+\alpha}{2} \frac{1}{n-1} \sum_{k=2}^{n}\left[\frac{2 c_{k}(\beta)}{k^{\frac{1}{\beta}}}\right]^{2 \alpha\left(\varphi^{n-k} \omega\right)-\alpha}\right\}
\end{aligned}
$$

Now consider the quantity

$$
\begin{aligned}
A_{n}(\omega) & :=\frac{1}{n-1} \sum_{k=2}^{n}\left[\frac{2 c_{k}(\alpha)}{k^{\frac{1}{\alpha}}}\right]^{\alpha\left(\varphi^{n-k} \omega\right)-\alpha} \\
& -\frac{1+\alpha}{2} \frac{1}{n-1} \sum_{k=2}^{n}\left[\frac{2 c_{k}(\beta)}{k^{\frac{1}{\beta}}}\right]^{2 \alpha\left(\varphi^{n-k} \omega\right)-\alpha}
\end{aligned}
$$


We estimate each sum in $A_{n}$ independently using the large deviations estimate detailed in Proposition 2.2.

For the first sum in Equation (2.2), using the substitution

$$
X_{k}(\omega)=\left[\frac{2 c_{k}(\alpha)}{k^{\frac{1}{\alpha}}}\right]^{\alpha\left(\varphi^{n-k} \omega\right)-\alpha}
$$

from which we compute

$$
E_{\omega}\left(X_{k}\right)=p_{1}+p_{2}\left[\frac{2 c_{k}(\alpha)}{k^{\frac{1}{\alpha}}}\right]^{\beta-\alpha}
$$

and using Proposition 2.2 and a positive value $t=t_{n}>0$ we obtain

$$
\begin{aligned}
\mathbb{P}\left\{\mid \frac{1}{n-1} \sum_{k=2}^{n}\left[\frac{2 c_{k}(\alpha)}{k^{\frac{1}{\alpha}}}\right]^{\alpha\left(\varphi^{n-k} \omega\right)-\alpha}\right. & \left.-\frac{1}{n-1} \sum_{k=2}^{n}\left(p_{1}+p_{2}\left[\frac{2 c_{k}(\alpha)}{k^{\frac{1}{\alpha}}}\right]^{\beta-\alpha}\right) \mid \geq t_{n}\right\} \\
& \leq \exp \left(-2(n-1) t_{n}^{2}\right) .
\end{aligned}
$$

If we choose $t_{n} \downarrow 0$ such that ${ }^{3} \sum_{n} \exp \left(-2(n-1) t_{n}^{2}\right)<\infty$ then by Borel-Cantelli, keeping in mind that $c_{k}(\alpha)$ is bounded, and

$$
\frac{1}{n-1} \sum_{k=2}^{n}\left(p_{1}+p_{2}\left[\frac{2 c_{k}(\alpha)}{k^{\frac{1}{\alpha}}}\right]^{\beta-\alpha}\right)=p_{1}+O\left(n^{1-\beta / \alpha}\right),
$$

we conclude that

$$
\frac{1}{n-1} \sum_{k=2}^{n}\left[\frac{2 c_{k}(\alpha)}{k^{\frac{1}{\alpha}}}\right]^{\alpha\left(\varphi^{n-k} \omega\right)-\alpha} \rightarrow p_{1}
$$

for almost every $\omega \in[0,1]$.

We can follow a similar argument for the second term in Equation (2.2). This time however,

$$
E_{\omega}\left(\frac{1+\alpha}{2} \frac{1}{n-1} \sum_{k=2}^{n}\left[\frac{2 c_{k}(\beta)}{k^{\frac{1}{\beta}}}\right]^{2 \alpha\left(\varphi^{n-k} \omega\right)-\alpha}\right)=O\left(n^{-\gamma}\right),
$$

where $\gamma=\min \{\alpha / \beta, 2-\alpha / \beta\}=\alpha / \beta>0$ since $\alpha<\beta$. Therefore we conclude that for almost every $\omega$

$$
\frac{1+\alpha}{2} \frac{1}{n-1} \sum_{k=2}^{n}\left[\frac{2 c_{k}(\beta)}{k^{\frac{1}{\beta}}}\right]^{2 \alpha\left(\varphi^{n-k} \omega\right)-\alpha} \rightarrow 0 .
$$

Combining Equations (2.4), and (2.5) shows that $A_{n} \rightarrow p_{1}$ almost everywhere.

It follows that almost surely (w.r.t. $\omega) \lim \inf _{n} \frac{1}{n\left[x_{n}(\omega)\right]^{\alpha}} \geq \alpha 2^{\alpha} p_{1}$. The statement of the lemma follows.

Lemma 2.4. There is a set $G_{2} \subseteq[0,1]$ of full measure such that for every $\omega \in G_{2}$ we have

$$
\liminf n^{\frac{1}{\alpha}} x_{n}(\omega) \geq\left[\alpha 2^{\alpha} p_{1}\right]^{-\frac{1}{\alpha}}=c(\alpha) p_{1}^{-\frac{1}{\alpha}} .
$$

\footnotetext{
${ }^{3} t_{n}=n^{-1 / 3}$ does the job, for example.
} 
Proof. Let $G_{1}$ be a set of full measure in $\omega$ for which convergence is obtained in Lemma 2.3. In particular, for every $\omega \in G_{1}$ there exists an $N=N(\omega)$ such that for all $n>N(\omega)$ we have

$$
x_{n}(\omega) \leq \frac{c(\alpha) p_{1}^{-\frac{1}{\alpha}}+1}{n^{\frac{1}{\alpha}}} .
$$

Now, starting with Equation (2.1), using the lower bound in Lemma 2.1, dividing by $n$, and assuming $\lfloor\sqrt{n}\rfloor \geq N(\omega)$ we get the following expression:

$$
\begin{aligned}
\frac{1}{n\left[x_{n}(\omega)\right]^{\alpha}} & \leq \frac{1}{n} 2^{\alpha}+\alpha 2^{\alpha} \frac{1}{n} \sum_{k=2}^{n}\left[2 x_{k}\left(\varphi^{n-k} \omega\right)\right]^{\alpha\left(\varphi^{n-k} \omega\right)-\alpha} \\
& \leq \frac{1}{n} 2^{\alpha}+\alpha 2^{\alpha} \frac{\lfloor\sqrt{n}\rfloor-1}{n} \frac{1}{\lfloor\sqrt{n}\rfloor-1} \sum_{k=2}^{\lfloor\sqrt{n}\rfloor}\left[2 x_{k}\left(\varphi^{n-k} \omega\right)\right]^{\alpha\left(\varphi^{n-k} \omega\right)-\alpha} \\
& +\frac{n-\lfloor\sqrt{n}\rfloor}{n} \frac{1}{n-\lfloor\sqrt{n}\rfloor} \sum_{\lfloor\sqrt{n}\rfloor+1}^{n}\left[2 \frac{c(\alpha) p^{-\frac{1}{\alpha}}+1}{n^{\frac{1}{\alpha}}}\right]^{\alpha\left(\varphi^{n-k} \omega\right)-\alpha}
\end{aligned}
$$

Now define, for any $\omega \in[0,1]$

$$
A_{n}^{\prime}(\omega):=\frac{1}{n-\lfloor\sqrt{n}\rfloor} \sum_{k=\lfloor\sqrt{n}\rfloor+1}^{n}\left[2 \frac{c(\alpha) p_{1}^{-\frac{1}{\alpha}}+1}{n^{\frac{1}{\alpha}}}\right]^{\alpha\left(\varphi^{n-k} \omega\right)-\alpha}
$$

Once again, application of the large deviation estimates in Proposition 2.2 combined with the direct calculation $E_{\omega}\left(A_{n}^{\prime}\right)=p_{1}+O\left(n^{1-\beta / \alpha}\right)$ shows that $A_{n}^{\prime} \rightarrow p_{1}$ for almost every $\omega$ in a set $G_{2}$ of full measure.

Finally, fix an arbitrary $\omega \in G_{1} \cap G_{2}$. Provided $n$ is large enough such that $\lfloor\sqrt{n}\rfloor \geq N(\omega)$ we estimate

$$
\frac{1}{n\left[x_{n}(\omega)\right]^{\alpha}} \leq \frac{1}{n} 2^{\alpha}+\alpha 2^{\alpha} \frac{\lfloor\sqrt{n}\rfloor}{n}+\alpha 2^{\alpha} \frac{n-\lfloor\sqrt{n}\rfloor}{n} A_{n}^{\prime}(\omega) .
$$

The right hand side of this expression converges to $\alpha 2^{\alpha} p_{1}$. It follows that for all $\omega \in G_{1} \cap G_{2}, \lim \inf n\left[x_{n}(\omega)\right]^{\alpha} \geq \alpha 2^{\alpha} p_{1}$. The lemma now follows by taking roots.

Lemmas 2.3 and 2.4 together give the almost sure convergence claimed in Theorem 1.1. To obtain the $L^{1}$ convergence we first observe

Lemma 2.5.

$$
\limsup _{n \rightarrow \infty} E_{\omega}\left(n^{\frac{1}{\alpha}} x_{n}(\omega)\right) \leq c(\alpha) p_{1}^{-\frac{1}{\alpha}} .
$$

Proof. Let $p_{0} \in\left(0, p_{1}\right)$. By Proposition 4.1 of [4] we have

$$
E_{\omega}\left(n^{\frac{1}{\alpha}} x_{n}(\omega)\right) \leq n^{\frac{1}{\alpha}} x_{\left\lfloor p_{0} n\right\rfloor}(\alpha)+n^{\frac{1}{\alpha}} \exp \left(-2 n\left(p_{1}-p_{0}\right)^{2}\right) .
$$

Let $\varepsilon>0$ be arbitrary. Since $x_{n}(\alpha)=c_{n}(\alpha) n^{-\frac{1}{n}}$, with $\inf c_{n}(\alpha)>0$ (see Equation (1.7)), for $n$ large enough, (2.7) implies

$$
E_{\omega}\left(n^{\frac{1}{\alpha}} x_{n}(\omega)\right) \leq(1+\varepsilon) n^{\frac{1}{\alpha}} x_{\left\lfloor p_{0} n\right\rfloor}(\alpha) .
$$

We also know from Equation (1.7) that

$$
x_{\left\lfloor p_{0} n\right\rfloor}(\alpha)=c_{\left\lfloor p_{0} n\right\rfloor}(\alpha)\left\lfloor p_{0} n\right\rfloor^{-\frac{1}{\alpha}},
$$


with $\lim _{n \rightarrow \infty} c_{\left\lfloor p_{0} n\right\rfloor}(\alpha)=c(\alpha)$. Consequently, by (2.8) and (2.9), we have

$E_{\omega}\left(n^{\frac{1}{\alpha}} x_{n}(\omega)\right) \leq(1+\varepsilon) n^{\frac{1}{\alpha}} c_{\left\lfloor p_{0} n\right\rfloor}(\alpha)\left\lfloor p_{0} n\right\rfloor^{-\frac{1}{\alpha}} \leq(1+\varepsilon) n^{\frac{1}{\alpha}} c_{\left\lfloor p_{0} n\right\rfloor}(\alpha) p_{0}-\frac{1}{\alpha}\left(n-\frac{1}{p_{0}}\right)^{-\frac{1}{\alpha}}$.

Thus,

$$
\limsup _{n \rightarrow \infty} E_{\omega}\left(n^{\frac{1}{\alpha}} x_{n}(\omega)\right) \leq(1+\varepsilon) c(\alpha) p_{0}^{-\frac{1}{\alpha}} .
$$

Since $p_{0}$ can be taken arbitrarily close to $p_{1}$, and $\varepsilon>0$ is arbitrary, (2.10) implies $\limsup _{n \rightarrow \infty} E_{\omega}\left(n^{\frac{1}{\alpha}} x_{n}(\omega)\right) \leq c(\alpha) p_{1}^{-\frac{1}{\alpha}}$.

Finally, Lemmas 2.3 and 2.4 combined with Lemma 2.5 give the required $L^{1}$ convergence due to the following elementary result.

Lemma 2.6. (Also Lemma 4.3 of Gouëzel [8]) Let $f_{n}$ be a sequence of integrable functions on a probability space, with $f_{n} \geq 0$ and $f_{n} \rightarrow f$ almost everywhere. Suppose that $E(f)<\infty$ and $\lim \sup E\left(f_{n}\right) \leq E(f)$. Then $E\left(\left|f_{n}-f\right|\right) \rightarrow 0$ (i.e. $f_{n} \rightarrow f$ in $L^{1}$ norm).

We include the proof for completeness.

Proof. Set $g_{n}:=\min \left\{f_{n}, f\right\}=\frac{1}{2}\left\{f_{n}+f-\left|f_{n}-f\right|\right\}$. Then $0 \leq g_{n} \leq f$ and $g_{n} \rightarrow f$ almost everywhere, so by dominated convergence $E\left(\left|g_{n}-f\right|\right) \rightarrow 0$. From this, we also see $E\left(g_{n}\right) \rightarrow E(f)$.

Now $\left|g_{n}-f_{n}\right|=f_{n}-g_{n} \geq 0$ so by the first part

$$
\begin{aligned}
\limsup E\left(\left|g_{n}-f_{n}\right|\right) & =\limsup E\left(f_{n}\right)-\liminf E\left(g_{n}\right) \\
& \leq E(f)-E(f) \\
& =0
\end{aligned}
$$

It follows that $\lim E\left(\left|g_{n}-f_{n}\right|\right)=0$ which completes the proof.

\section{Application to limit theorems}

In this section we apply Theorem 1.1 to establish limit theorems for the Birkhoff sums $S_{n} f(x, \omega)$ in (1.10) when $f: I \times I \rightarrow \mathbb{R}$ is Hölder-continous and $\int f d \nu=0$. Here, $\nu=h d m \times d m$ is the absolutely continuous invariant measure for $S$.

We first observe that for $0<\alpha<\beta<1$ the induced map $\left(S^{R}, \Delta_{0}\right)$ is GibbsMarkov for the return-time partition $\Delta_{0, i}$ (see Aaronson [1] or Aaronson and Denker [2] for a definition of Gibbs-Markov). The required expansion and distortion estimates are derived in [4] with respect to the metric $d\left(z_{1}, z_{2}\right):=\theta^{s\left(z_{1}, z_{2}\right)}$, for a suitable constant $0<\theta<1, z_{1}=\left(x_{1}, \omega_{1}\right), z_{2}=\left(x_{2}, \omega_{2}\right)$ and $s\left(z_{1}, z_{2}\right)$ being the usual separation time of two points in $\Delta_{0}$ with respect to the return partition.

Next, we establish local Lipschitz regularity for the density $h$ away from the fixed point.

Lemma 3.1. For $0<\alpha<\beta<1$, $h$ has a version that is Lipschitz on any compact subset of $(0,1] \times[0,1]$.

Proof. By Theorem 5.2 of [5], we know that the unique invariant density of $S$ is of the form $h=g \times \mathbf{1}$ where $P_{T} g=g, P_{T}$ being the transfer operator associated with the random map $T$. Thus, it is enough to show that $g$ is Lipschitz on compact subsets of $(0,1]$. We prove this fact by studying the action of $P_{T}$ on a suitable cone. 
Let $\mathcal{B}$ denote the set of integrable and $C^{1}$ functions on $(0,1]$. For $a>0$, define a cone $\mathcal{C}_{a}$ by

$$
\mathcal{C}_{a}=\left\{f \in \mathcal{B} \mid f \geq 0, f \text { decreasing, } \int_{0}^{x} f d \lambda \leq a x^{1-\beta} \int_{0}^{1} f\right\} .
$$

Let $a_{*}=\frac{4}{1-\beta}$. For $a \geq a_{*}$, it is well known that under the action of $P_{T_{\beta}}$, the transfer operator associated with the map $T_{\beta}, \mathcal{C}_{a}$ is invariant. Moreover, since $\alpha<\beta$ the same is true for $P_{T_{\alpha}}$. Since $P_{T}$ is a convex combination of $P_{T_{\alpha}}$ and $P_{T_{\beta}}$, we conclude that $\mathcal{C}_{a}$ is invariant under the action of $P_{T}$. Consequently, $P_{T}$ has a fixed point in $\mathcal{C}_{a}$ (an invariant density), which we denoted by $g$. Note that $g$ is Lipschitz on any compact subset of $(0,1]$ by the properties of $\mathcal{C}_{a}$.

From this point on we assume $h$ is the (unique) Lipschitz version assured by this lemma since this plays a key role in some of the estimates to follow.

Set $A:=\frac{1}{2} c(\alpha) p_{1}^{-\frac{1}{\alpha}} E_{\omega}\left(h\left(\frac{1}{2}, \omega\right)\right)$. Let $\mathcal{N}\left(0, \sigma^{2}\right)$ denote the normal distribution with mean zero and variance $\sigma^{2}$.

Theorem 3.2. Let $0<\alpha<\beta<1$. Let $f: I \times I \rightarrow \mathbb{R}$ be a Hölder continuous function satisfying $\int f d \nu=0$. Set $c:=E_{\omega}(f(0, \omega))$. Then

(1) If $\alpha<\frac{1}{2}$, there exists $\sigma^{2} \geq 0$ such that

$$
\frac{1}{\sqrt{n}} S_{n} f \rightarrow \mathcal{N}\left(0, \sigma^{2}\right)
$$

(2) If $\frac{1}{2} \leq \alpha<1$ and $c=0$, suppose there exists a $\gamma>\frac{\beta}{\alpha}\left(\alpha-\frac{1}{2}\right)$ such that $|f(x, \omega)-f(0, \omega)| \leq C_{f} x^{\gamma}$. Then there exists $\sigma^{2} \geq 0$ such that

$$
\frac{1}{\sqrt{n}} S_{n} f \rightarrow \mathcal{N}\left(0, \sigma^{2}\right) .
$$

(3) If $\alpha=\frac{1}{2}$ and $c \neq 0$ then $S_{n} f / \sqrt{c^{2} A n \ln n} \rightarrow \mathcal{N}(0,1)$.

(4) If $\frac{1}{2}<\alpha<1$ and $c \neq 0$ then $S_{n} f / n^{\alpha} \rightarrow Z$ where the random variable $Z$ has characteristic function given by

$$
E(\exp (i t Z))=\exp \left\{-A|c|^{\frac{1}{\alpha}} \Gamma\left(1-\frac{1}{\alpha}\right) \cos (\pi / 2 \alpha)|t|^{\frac{1}{\alpha}}(1-i \operatorname{sgn}(c t) \tan (\pi / 2 \alpha))\right\} \text {. }
$$

The proof depends on a number of careful estimates using Theorem 1.1 that can be proved in an analogous way to corresponding calculations in Gouëzel [8].

Lemma 3.3. We have $\nu(R>n) \sim n^{-1 / \alpha} A$.

Notation 3.4. For $f:[0,1] \times[0,1] \rightarrow \mathbb{R}$ and $(x, \omega) \in \Delta_{0}$ define

$$
f_{\Delta_{0}}(x, \omega):=\sum_{k=0}^{R(x, \omega)-1} f\left(S^{k}(x, \omega)\right) .
$$

Lemma 3.5. Let $f$ be Hölder on $[0,1] \times[0,1]$. If $0<\alpha<1 / 2$, then $f_{\Delta_{0}} \in$ $L^{2}\left(\Delta_{0}, d \nu\right)$.

Lemma 3.6. Suppose $f: X \rightarrow \mathbb{R}$ is Hölder continuous with $E_{\omega}(f(0, \omega))=0$. Suppose there are constants $0<\gamma<\beta$ and $C_{f}<\infty$ such that, uniformly in $\omega$, for all $x \in[0,1]$

$$
|f(x, \omega)-f(0, \omega)| \leq C_{f} x^{\gamma} .
$$

Let $1 \leq p<\min \left\{2 / \alpha, 1 / \alpha\left(1-\frac{\gamma}{\beta}\right)\right\}$. Then $f_{\Delta_{0}} \in L^{p}\left(\Delta_{0}, \nu\right)$. 
We are going to work with a family of metrics based on separation time. For $2 \leq \lambda<\infty$, the expansion constant for $S^{R}$ and any $0<\theta<1$ define

$$
d_{\lambda^{-\theta}}\left(z_{1}, z_{2}\right):=\lambda^{-\theta s\left(z_{1}, z_{2}\right)} .
$$

It follows that with respect to $d_{\lambda^{-\theta}}$, the expansion constant of $S^{R}$ is at least $\lambda^{\theta}>1$, and that $S^{R}$ is Gibbs-Markov for this metric and the partition $\Delta_{0, i}^{s}$.

Let $0<\theta<1$ be the Hölder exponent of $f$. For each $n, s$ let $D f_{\Delta_{0}}\left(\Delta_{0, n}^{s}\right)$ denote the Lipschitz constant of $f_{\Delta_{0}}$ restricted to the subset $\Delta_{0, n}^{s}$ and computed with respect to the metric $d_{\lambda^{-\theta}}$.

\section{Lemma 3.7.}

$$
\sum_{n, s} \nu\left(\Delta_{0, n}^{s}\right) D f_{\Delta_{0}}\left(\Delta_{0, n}^{s}\right) \leq C \sum_{n} \nu\{R=n\} n<\infty .
$$

3.1. Proof of Theorem 3.2. We are going to use Theorem 3.1 of Gouëzel [8]. The basic finite expectation condition (Equation (18) in [8]) is given in our setting by Lemma 3.7.

Assume first that $\alpha<\frac{1}{2}$. Then by Lemma 3.5 we know that $f_{\Delta_{0}} \in L^{2}\left(\Delta_{0}, \nu\right)$. Also the return time function $R \in L^{2}$ since $R=g_{\Delta_{0}}$ with $g \equiv 1$, to which Lemma 3.5 also applies. This is the setting of the first case of Theorem 3.1, so we obtain the central limit theorem in (1).

Next we consider $1 / 2 \leq \alpha<1$ and $c=0$. Now Lemma 3.6 with conditions given on $\gamma$ shows that $f_{\Delta_{0}} \in L^{2}$ and the estimate in Lemma 3.3 shows that

$$
\nu\{R>n\}=n^{-\frac{1}{\alpha}} A(n) \sim n^{-\frac{1}{\alpha}} A .
$$

For $z \in(0, \infty)$ we get $\nu\{R>z\}=\lceil z\rceil^{-\frac{1}{\alpha}} A(\lceil z\rceil)$. Set $\Lambda(z):=(\lceil z\rceil / z)^{-\frac{1}{\alpha}} A(\lceil z\rceil)$. Note that $z \rightarrow \Lambda(z)$ is slowly varying, $\Lambda(z) \sim A$ and $\nu\{R>z\}=z^{-\frac{1}{\alpha}} \Lambda(z)$. The second sub-condition in the first case of Theorem 3.1 [8] are therefore satisfied with $L:=\Lambda$ and we again get a central limit theorem in (2).

The last two cases require a more detailed estimate. Assume $\frac{1}{2} \leq \alpha<1$. Set $g \equiv c$ and note that on $g_{\Delta_{0}}(x, \omega)=c n \Longleftrightarrow R(x, \omega)=n$, so $\nu\left\{\left|g_{\Delta_{0}}\right|>z\right\} \sim$ $|c|^{\frac{1}{\alpha}} z^{-\frac{1}{\alpha}} \Lambda(z)$ according to the previous calculation. The function $j=f-g$ has the same regularity as $f$ and satisfies $E_{\omega}(j(0, \omega))=0$. We next show that $\nu\left\{\left|j_{\Delta_{0}}\right|>\right.$ $z\}=o\left(z^{-\frac{1}{\alpha}}\right)$. Applying Lemma 3.6 to $j$, we obtain $p>\frac{1}{\alpha}$ such that $j_{\Delta_{0}} \in L^{p}\left(\Delta_{0}\right)$. It follows that

$$
\nu\left\{\left|j_{\Delta_{0}}\right|>z\right\} \leq \int\left(\left|j_{\Delta_{0}}\right| / z\right)^{p} d \nu=C z^{-p}=o\left(z^{-\frac{1}{\alpha}}\right) .
$$

The elementary decomposition

$$
\left\{g_{\Delta_{0}}>z(1+\epsilon)\right\} \cap\left\{\left|j_{\Delta_{0}}\right| \leq \epsilon z\right\} \subseteq\left\{f_{\Delta_{0}}>z\right\} \subseteq\left\{g_{\Delta_{0}}>z(1-\epsilon)\right\} \cup\left\{\left|j_{\Delta_{0}}\right|>\epsilon z\right\}
$$

implies

$$
\begin{aligned}
\nu\left\{g_{\Delta_{0}}>z(1+\epsilon)\right\}-\nu\left\{\left|j_{\Delta_{0}}\right|>\epsilon z\right\} & \leq \nu\left\{f_{\Delta_{0}}>z\right\} \\
& \leq \nu\left\{g_{\Delta_{0}}>z(1-\epsilon)\right\}+\nu\left\{\left|j_{\Delta_{0}}\right|>\epsilon z\right\} .
\end{aligned}
$$

Now consider the case $\alpha=\frac{1}{2}$ in (3). Assume $c>0$ and use the asymptotic estimates on the upper and lower bounds in Equation (3.1) to obtain $\nu\left\{f_{\Delta_{0}}>z\right\} \sim$ $z^{-2}\left(c^{2} A+o(1)\right)$. On the other hand, if $c<0$ then $g<0$ and $\left\{g_{\Delta_{0}}>z(1 \pm \epsilon)\right\}=$ $\emptyset$, so $\nu\left\{f_{\Delta_{0}}>z\right\} \leq \nu\left\{\left|j_{\Delta_{0}}\right|>\epsilon z\right\} \sim z^{-2} o(1)$. Combining these two estimates yields $\nu\left\{\left|f_{\Delta_{0}}\right|>z\right\} \sim z^{-2}\left(c^{2} A+o(1)\right):=z^{-2} l(z)$, independent of the sign of 
$c$. The only difference is that for $c>0$ the tail distribution is heavy for postive $z$ while for $c<0$ it is heavy for negative $z$. We have already established that $\nu\{R>z\} \sim z^{-2} A=z^{-2}\left(1 / c^{2}\right) l(z)$. We can now apply the third case of Theorem 3.1 [8] with $L(z)=2 c^{2} A \int_{1}^{z} \frac{1}{u} d u=2 c^{2} A \ln z$ (unbounded and slowly varying) and $B_{n}:=\sqrt{c^{2} A n \ln n}$, whereby $n L\left(B_{n}\right) \sim B_{n}^{2}$ as required.

For the final case, when $\frac{1}{2}<\alpha<1$ we return to the estimate in Equation (3.1) and again, we first assume $c>0$, so that $g>0$. For $z>0$ the asymptotic estimates yield

$$
\nu\left\{f_{\Delta_{0}}>z\right\} \sim z^{-\frac{1}{\alpha}}\left(c^{\frac{1}{\alpha}} A+o(1)\right)
$$

while we have already established that $\nu\{R>z\} \sim z^{-\frac{1}{\alpha}} A$. On the other hand,

$$
\nu\left\{f_{\Delta_{0}}<-z\right\} \leq \nu\left\{\left|j_{\Delta_{0}}\right|>\epsilon z\right\}=o\left(z^{-\frac{1}{\alpha}}\right) .
$$

We can therefore apply the last case in Theorem 3.1, [8], setting $c_{1}=c^{\frac{1}{\alpha}} A, c_{2}=0$, $c_{3}=A, L \equiv 1$ and $B_{n}:=n^{\alpha}$. In the case $c<0$ we simply exchange the values of $c_{1}$ and $c_{2}$. Putting this together, the theorem gives an asymptotic stable law with characteristic function

$$
\exp \left[-c^{\frac{1}{\alpha}} A \Gamma\left(1-\frac{1}{\alpha}\right) \cos \left(\frac{\pi}{2 \alpha}\right)|t|^{\frac{1}{\alpha}}\left(1-i \operatorname{sgn}(c) \operatorname{sgn}(t) \tan \left(\frac{\pi}{2 \alpha}\right)\right)\right],
$$

which is case (4) in our theorem. This completes the proof.

\section{Application to CORRElation asymptotics for infinite measure PRESERVING RANDOM SYSTEMS}

In this section we use arguments analogous to Theorem 1.1 to study the asymptotics of the transfer operator associated with the skew product of a random version of the maps studied by Gaspard and Wang in [6]. In particular, we will consider linearizations ${ }^{4}$ of random LSV transformations as follows. For a parameter value $1 \leq \alpha<\infty$, define the sequence $\xi_{k}(\alpha)=\frac{1}{2(1+k)^{\frac{1}{\alpha}}} \in\left(0, \frac{1}{2}\right], k=0,1,2, \ldots$ and $\xi_{-1}(\alpha)=1$. Define the map $T_{\alpha}: I \rightarrow I$ by

$T_{\alpha}(x)= \begin{cases}\frac{\xi_{k-2}(\alpha)-\xi_{k-1}(\alpha)}{\xi_{k-1}(\alpha)-\xi_{k}(\alpha)}\left(x-\xi_{k}(\alpha)\right)+\xi_{k-1}(\alpha), \text { for } x \in\left(\xi_{k}(\alpha), \xi_{k-1}(\alpha)\right], k=1,2, \ldots \\ 2 x-1, & \text { for } x \in\left(\frac{1}{2}, 1\right] .\end{cases}$

$T_{\alpha}$ is a piecewise affine version of the Pomeau-Manneville map with parameter $\alpha$. We now fix two parameters $1 \leq \alpha<\beta<\infty$ and consider the linearized random LSV transformation defined as follows:

$$
T=\left\{T_{\alpha}(x), T_{\beta}(x) ; p_{1}, p_{2}\right\}, \text { where }
$$

$p_{1}, p_{2}>0$ and $p_{2}=1-p_{1}$. Let $S$ denote the associated skew product which is defined in the same way as (1.1). As in Subsection 1.2, we induce $S$ on $\Delta_{0}$. Thus, by our theorem, we can apply Theorem 1.4 of $^{5}$ [9] to obtain asymptotics of $\mathcal{L}_{S}^{n}$. In particular, we obtain the following theorem.

\footnotetext{
${ }^{4}$ We consider a linearized version because for general random LSV transformations we were able to prove bounded distortion only when $0<\alpha \leq \beta \leq 1$. See [4] for the result and a discussion on distortion.

${ }^{5}$ For more general observables, one can use the result of [13]. However, one would lose uniform convergence for all parameters. See $[13,9]$ for a discussion.
} 
Theorem 4.1. Let $S$ be the skew product defined using (4.1) with $1 \leq \alpha<\beta<\infty$. The following hold:

(1) $S$ admits a unique absolutely continuous invariant infinite ( $\sigma$-finite) measure $\nu$;

(2) Let $\mathcal{L}_{\nu}$ be the transfer operator associated with $S$ with respect to the invariant measure $\nu$. For $\alpha>1$, let $f$ be a Lipschitz function supported on $\Delta_{0}$. Then

$$
\lim _{n \rightarrow \infty}\left\|n^{1-\frac{1}{\alpha}} \mathbf{1}_{\Delta_{0}} \mathcal{L}_{\nu}^{n} f-c \int_{\Delta_{0}} f\right\|_{\infty}=0
$$

where $c$ is a constant independent of $f$. In particular, if $g \in L^{1}\left(\Delta_{0}\right)$, we have

$$
\lim _{n \rightarrow \infty} n^{1-\frac{1}{\alpha}} \int_{\Delta_{0}} f \cdot g \circ S^{n}=c \int_{\Delta_{0}} f \int_{\Delta_{0}} g .
$$

(3) For $\alpha=1$, and $f$ a Lipschitz function supported on $\Delta_{0}$, we obtain the same results as in (2) with normalizing sequence $\ln n$ instead of $n^{1-\frac{1}{\alpha}}$.

Proof. We first notice that induced skew product $S^{R}: \Delta_{0} \rightarrow \Delta_{0}$ is piecewise affine and onto. In particular, it satisfies the assumptions of Aaronson-Denker [2]. Thus, it has a unique absolutely continuous invariant probability measure $\nu_{\Delta_{0}}$ whose density $h_{\Delta_{0}} \in \mathcal{B}$, where $\mathcal{B}$ is the space of Lipschitz functions on $\Delta_{0}$, the system $\left(S^{R}, \nu_{\Delta_{0}}\right)$ is mixing and the associated transfer operator $\mathcal{L}_{S^{R}}$, with respect to $\nu_{\Delta_{0}}$, has a spectral gap on $\mathcal{B}$. The $S$-invariant measure, $\nu$, is defined using $\nu_{\Delta_{0}}$. The fact that the measure $\nu$ is infinite ( $\sigma$-finite) follows from Theorem 1.1 since $\alpha \geq 1$. We normalize $\nu$ so that $\nu\left(\Delta_{0}\right)=1$. To prove (2), we apply Gouëzel [9] Theorem 1.4. For $f \in \mathcal{B}$ define

$$
R_{n} f:=1_{\Delta_{0}} \mathcal{L}_{\nu}^{n}\left(1_{\{R=n\}} f\right),
$$

where $\mathcal{L}_{\nu}$ is the transfer operator associated with $S$ with respect to the invariant measure $\nu$. Using (4.2) and $\mathcal{L}_{S^{R}}$, we get

$$
\mathcal{L}_{S^{R}}(f)=\sum_{n \geq 1} \mathcal{L}_{\nu}^{n}\left(1_{\{R=n\}} f\right) .
$$

The spectral properties of $\mathcal{L}_{S^{R}}$ imply that $\left(R_{n}\right)_{n \geq 1}$ is an aperiodic renewal sequence of operators (see Sarig [16] for the definition). We still need to check:

- $\nu(\{R>n\}) \sim n^{-\frac{1}{\alpha}} l(n)$, where $l$ is slowly varying function;

- $\exists C>0$ such that $\left\|R_{n}\right\|_{\text {Lip }} \leq C n^{-\frac{1}{\alpha}-1}$.

The first condition follows from Lemma 3.3. For the second one, using (8) on page 649 of [16], $\exists C>0$ such that

$$
\left\|R_{n}\right\|_{\text {Lip }} \leq C \nu(\{R=n\}) .
$$

Thus, Lemma 3.3 completes the proof of (2). For (3) the proof is essentially the same as in (2) but using part (a) of Theorem 1.1 in [13].

Remark 4.2. When $0<\alpha<1$ and $\alpha<\beta<\infty$ we can use the arguments from Theorem 1.2 to prove that the skew product of linearized LSV maps has a finite invariant measure over the full range of $0<\beta<\infty$, even though the GaspardWang maps have only infinite absolutely continuous invariant measures when $1 \leq$ $\beta<\infty$. The required bounded distortion condition is automatically satisfied for 
the Gaspard-Wang family. Asymptotic estimates as in Theorem 1.1 lead to decay of correlation results as in Theorem 1.2 and limit laws as in Theorem 3.2.

Acknowledgment. The authors thank Ian Melbourne for bringing the piecewise affine maps in reference [6] to their attention.

\section{REFERENCES}

1. Aaronson, J. An introduction to infinite ergodic thoery. Mathematical Surveys and Monographs 1, 50. Amer. Math. Soc, Providence RI, 1997.

2. Aaronson, J. and Denker, M. Local limit theorems for partial sums of stationary sequences generated by Gibbs-Markov maps. Stoch. \& Dynam. 1 no. 2 (2001), 193-237.

3. Ayyer, A. Liverani, C. and Stenlund, M. Quenched CLT for random toral automorphisms. Discrete Contin. Dyn. Syst. 24, no. 2 (2009), 331-348.

4. Bahsoun, W.; Bose, C.; Duan, Y. Decay of correlation for random intermittent maps. Nonlinearity. 27 (2014) 1543-1554.

5. Bahsoun, W.; Bose, C.; Quas, A., Deterministic representation for position dependent random maps. Discrete Contin. Dyn. Syst. 22 (2008), 529-540.

6. Gaspard, P.; Wang, X.-J. Sporadicity: between periodic and chaotic dynamical behaviors. Proc. Nat. Acad. Sci. U.S.A. 85 (1988), no. 13, 4591-4595.

7. Gouëzel, S., Sharp polynomial estimates for the decay of correlations. Israel J. Math. 139 (2004), 29-65.

8. Gouëzel, S., Statistical properties of a skew product with a curve of neutral points. Ergodic Theory Dynam. Systems 27 (2007), 123-151.

9. Gouëzel, S., Correlation asymptotics from large deviations in dynamical systems with infinite measure. Colloq. Math. 125 (2011), no. 2, 193-212.

10. Hoeffding, W., Probability inequalities for sums of bounded random variables J. Amer. Stat. Soc. 58 \# 301 (1963), 13-30.

11. Leppänen, J. and Stenlund, M. Quasistatic dynamics with intermittency. Available on http://arxiv.org/abs/1510.02748

12. Liverani, C., Saussol, B. and Vaienti, S., A probabilistic approach to intermittency, Ergodic Theory Dynam. Systems 19 (1999), 671-685.

13. Melbourne, I. and Terhesiu, D. Operator renewal theory and mixing rates for dynamical systems with infinite measure. Invent. Math. 189 (2012), no. 1, 61-110.

14. Pianigiani, G. First return map and invariant measures. Israel J. Math. 35 (1980), 32-48.

15. Pomeau, Y. and Manneville, P. Intermittent transition to turbulence in dissipative dynamical systems. Comm. Math. Phys. (74 (1980) 189-197.

16. Sarig, O. Subexponential decay of correlations. Invent. Math. 150 (2002), 629-653.

17. Young, L-S., Recurrence times and rates of mixing. Israel J. Math., 110 (1999), 153-188.

18. Zweimüller, R. Ergodic structures and invariant densities for non-Markovian inteval maps with indifferent fixed points. Nonlinearity. 11 (1998), 1263-1276.

19. Zweimüller, R. Mixing limit theorems for ergodic transformations. J. Theor. Prob. 20 (2007), 1059-1071.

Department of Mathematical Sciences, Loughborough University, Loughborough, LEICESTERSHIRE, LE11 3TU, UK

E-mail address: $†$ W.Bahsoun@lboro.ac.uk

Department of Mathematics and Statistics, University of Victoria, PO BOX 3045

STN CSC, Victoria, B.C., V8W 3R4, Canada

E-mail address: * cbose@uvic.ca 